\title{
GROUPS WHICH DO NOT ADMIT GHOSTS
}

\author{
SUNIL K. CHEBOLU, J. DANIEL CHRISTENSEN, AND JÁN MINÁČ
}

(Communicated by Paul Goerss)

\begin{abstract}
A ghost in the stable module category of a group $G$ is a map between representations of $G$ that is invisible to Tate cohomology. We show that the only non-trivial finite $p$-groups whose stable module categories have no non-trivial ghosts are the cyclic groups $C_{2}$ and $C_{3}$. We compare this to the situation in the derived category of a commutative ring. We also determine for which groups $G$ the second power of the Jacobson radical of $k G$ is stably isomorphic to a suspension of $k$.
\end{abstract}

\section{INTRODUCTION}

Let $G$ be a $p$-group and let $k$ be a field of characteristic $p$. A natural home for the cohomology of modular representations of $G$ is the stable module category $\operatorname{StMod}(k G)$ of $G$. It is the category obtained from the category of left $k G$-modules by killing the projectives. It has the structure of a tensor triangulated category with the trivial representation $k$ as the unit object and $\Omega$ as the loop (desuspension) functor. The space of morphisms from $M$ to $N$ in $\operatorname{StMod}(k G)$ is denoted $\underline{\operatorname{Hom}}_{k G}(M, N)$ and consists of the $k G$-module homomorphisms modulo those that factor through a projective module. Note that a map of $k G$-modules that factors through a projective clearly induces the zero map in Tate cohomology. A natural question is whether the converse is true. Namely, if $f: M \rightarrow N$ is a map of $k G$-modules such that the induced map in Tate cohomology

$$
\underline{\operatorname{Hom}}_{k G}\left(\Omega^{*} k, M\right) \longrightarrow \underline{\operatorname{Hom}}_{k G}\left(\Omega^{*} k, N\right)
$$

is trivial, then does $f$ factor through a projective module? Equivalently, is every such $f$ trivial in the stable module category? In this paper we investigate the groups $G$ for which the above question always has an affirmative answer. Our main theorem states:

Theorem 1.1. Let $G$ be a non-trivial finite p-group and let $k$ be a field of characteristic $p$. All maps which are trivial in Tate cohomology factor through a projective $k G$-module if and only if $G$ is either $C_{2}$ or $C_{3}$.

A map between $k G$-modules is said to be a ghost if the induced map in Tate cohomology $\underline{\operatorname{Hom}}_{k G}\left(\Omega^{*} k,-\right)$ is trivial. Using this terminology, the main theorem says that the only finite $p$-groups for which all ghosts are trivial in the stable module

Received by the editors October 13, 2006, and, in revised form, January 2, 2007.

2000 Mathematics Subject Classification. Primary 20C20, 20J06; Secondary 55P42.

Key words and phrases. Ghost map, stable module category, derived category, Jennings' theorem, generating hypothesis. 
category are $C_{2}, C_{3}$ and the trivial group. It is interesting and surprising to note that the answer is so simple.

We now explain the strategy of the proof. We construct a (weakly) universal ghost out of a given $k G$-module $M$, and from this, we deduce that all ghosts out of $M$ vanish if and only if $M$ is a retract of a direct sum of suspensions of $k$. In particular, when $M$ is a finite-dimensional non-projective indecomposable module in $\operatorname{StMod}(k G)$, all ghosts out of $M$ vanish if and only if $M \cong \Omega^{i} k$ in $\operatorname{StMod}(k G)$ for some $i$. Therefore, we determine the finite $p$-groups $G$ which admit a non-projective indecomposable $k G$-module that is not stably isomorphic to any $\Omega^{i} k$. A formula of Jennings [7], which computes the nilpotency index of the Jacobson radical of $k G$, plays an important role in this investigation.

The proof (sketched above) of our main result yields some interesting additional results. For instance, Proposition 3.7 characterises finite $p$-groups $G$ for which the second power of the Jacobson radical of $k G$ is isomorphic in the stable module category to a suspension of $k$. In addition, the formal material in Section 2 has implications in other settings, as we illustrate in Section 4.

We now explain how we were led to these results. An old conjecture of Peter Freyd [6] in homotopy theory called the generating hypothesis $(\mathrm{GH})$ claims that a map $\phi: X \rightarrow Y$ between finite spectra that induces the zero map on stable homotopy groups is null-homotopic. It is one of the most important unsolved problems in stable homotopy theory. In order to gain some insight into this deep problem, it is natural to examine its analogues in algebraic settings such as the derived category of a commutative ring and the stable module category of a finite group. The GH in the derived category $D(R)$ of a ring $R$ is the statement that a map $\phi: X \rightarrow Y$ between perfect complexes that induces the zero map in homology is chain-homotopic to the zero map. Keir Lockridge [8] showed that the GH holds in the derived category of a commutative ring $R$ if and only if $R$ is a von Neumann regular ring.

In the stable module category, the GH is the statement that a map $\phi: M \rightarrow N$ between finite-dimensional $k G$-modules is trivial in $\operatorname{StMod}(k G)$ if the induced map in Tate cohomology is trivial. This paper takes a first step towards the GH by studying the variant in which the modules are not assumed to be finite-dimensional. The theorem above implies that the GH is true for $C_{2}$ and $C_{3}$, but does not answer the question for other groups, since it does not guarantee the existence of ghosts between finite-dimensional modules. Somewhat surprisingly, this variant of the GH turns out to be equivalent to the GH. Indeed, motivated by the main result of this paper, we have shown in joint work with Dave Benson [3] that the GH holds for a non-trivial finite $p$-group $G$ if and only if $G$ is either $C_{2}$ or $C_{3}$. This result can be used to deduce the main theorem stated above. However, the methods used in the two papers are completely different. In this paper, we use techniques inspired from homotopy theory and classical group theory, while the techniques in [3] are more representation theoretic, relying heavily on the induction and restriction. Moreover, we should emphasise that the methods in [3] do not give the additional results mentioned above.

We end the introduction by posing a riddle to the reader: Which finite $p$-groups are like a finite product of fields? We solve this riddle in the last section using a 
result of Lockridge, which is an analogue of our main theorem for the derived category of a commutative ring. Thus the riddle sets a context for our main theorem, both in representation theory and commutative algebra.

\section{Ghosts in triangulated Categories}

Let $\mathcal{T}$ be a triangulated category which admits arbitrary coproducts and let $S$ be a distinguished object. (If $\mathcal{T}$ is tensor triangulated, we always take $S$ to be the unit object of $\mathcal{T}$.) If $X$ and $Y$ are objects in $\mathcal{T}$, then $[X, Y]_{*}$ will denote the graded abelian group of maps from $X$ to $Y$, and $\pi_{*}(X)$ will stand for $[S, X]_{*}$. A map $\phi: M \rightarrow N$ in $\mathcal{T}$ is a ghost if the induced map

$$
\pi_{*}(M) \stackrel{\phi_{*}}{\longrightarrow} \pi_{*}(N)
$$

is trivial. We say that $\phi: M \rightarrow N$ is a universal ghost if $\phi$ is a ghost and if every ghost out of $M$ factors through $\phi$. (Such a map should technically be called weakly universal because we don't assume the factorisation to be unique.) We begin by showing the existence of a universal ghost out of any given object in $\mathcal{T}$. This technique is well-known in homotopy theory, but we include the details in this section to keep the paper self-contained.

Let $M$ be an object in $\mathcal{T}$. We assemble all the homogeneous elements of $\pi_{*}(M)$ into a map

$$
\coprod_{\eta \in \pi_{*}(X)} \Sigma^{|\eta|} S \longrightarrow M
$$

from a coproduct of suspensions of the unit object, where $|\eta|$ is the degree of $\eta$. Completing this map to an exact triangle in $\mathcal{T}$, we get

$$
\coprod_{\eta \in \pi_{*}(X)} \Sigma^{|\eta|} S \longrightarrow M \stackrel{\Phi_{M}}{\longrightarrow} U_{M} .
$$

Proposition 2.1. The map $\Phi_{M}: M \rightarrow U_{M}$ is a universal ghost out of $M$.

Proof. It is clear from its construction that $\Phi_{M}$ is a ghost. Now suppose $f: M \rightarrow N$ is any ghost. Then for each $\eta$ in $\pi_{*}(M)$, the composite

$$
\Sigma^{|\eta|} S \stackrel{\eta}{\longrightarrow} M \stackrel{f}{\longrightarrow} N
$$

is null. Therefore, so is the composite

$$
\coprod_{\eta \in \pi_{*}(M)} \Sigma^{|\eta|} S \longrightarrow M \stackrel{f}{\longrightarrow} N .
$$

Now (2.1) is an exact triangle and therefore $f$ factors through $\Phi_{M}$.

Proposition 2.1 essentially says that ghosts form part of a projective class. See [5] for more details.

Recall that an object $C$ in $\mathcal{T}$ is said to be compact if the natural map

$$
\bigoplus_{\alpha}\left[C, X_{\alpha}\right] \longrightarrow\left[C, \coprod_{\alpha} X_{\alpha}\right]
$$

is an isomorphism for all set-indexed collections of objects $X_{\alpha}$ in $\mathcal{T}$. An object $X$ in $\mathcal{T}$ is said to be indecomposable if $X \neq 0$ and a decomposition $X \cong A \amalg B$ in $\mathcal{T}$ implies that either $A$ or $B$ is the zero object.

We say that $\mathcal{T}$ has the Krull-Schmidt property if the following two conditions hold: 
- Each compact object in $\mathcal{T}$ can be decomposed uniquely into indecomposable objects.

- The distinguished object $S$ in $\mathcal{T}$ is compact and indecomposable.

Our next proposition characterises the objects in $\mathcal{T}$ out of which all ghosts vanish.

Proposition 2.2. Let $\mathcal{T}$ be a triangulated category which admits arbitrary coproducts and let $S$ be a distinguished object. Then the following are equivalent for an object $M$ in $\mathcal{T}$ :

(1) All ghosts out of $M$ are trivial.

(2) The universal ghost $\Phi_{M}: M \rightarrow U_{M}$ is trivial.

(3) $M$ is a retract of a coproduct of suspensions of $S$.

Moreover, if $M$ is compact, then (3) is equivalent to:

$\left(3^{\prime}\right) M$ is a retract of a finite coproduct of suspensions of $S$.

If $M$ is compact and $\mathcal{T}$ has the Krull-Schmidt property, then (3) is equivalent to:

$\left(3^{\prime \prime}\right) M$ is a finite coproduct of suspensions of $S$.

Proof. (1) $\Rightarrow(2)$ is trivial, for $\Phi_{M}: M \rightarrow U_{M}$ is itself a ghost. Now to see that (2) implies (3), suppose the universal ghost $\Phi_{M}$ is trivial. That means that (2.1) is a split triangle. In particular, $M$ is a retract of $\amalg \Sigma^{i} S$. So there exists a map $M \stackrel{j}{\longrightarrow} \amalg \Sigma^{i} S$ such that the composite

$$
M \stackrel{j}{\longrightarrow} \coprod_{\pi_{*}(M)} \Sigma^{i} S \longrightarrow M
$$

is the identity in $\mathcal{T}$. Now if $M$ is compact, then $j$ factors through a finite coproduct. Therefore $M$ is a retract of a finite coproduct of suspensions of $S$. If $\mathcal{T}$ has the Krull-Schmidt property, then it follows that $M$ is a finite coproduct of suspensions of $S$. Finally $(3) \Rightarrow(1)$ is clear.

For reference we record the following corollaries which are immediate from Proposition 2.2 .

Corollary 2.3. Let $\mathcal{T}$ be a triangulated category which admits arbitrary coproducts and which has the Krull-Schmidt property, and let $S$ be a distinguished object. If $M$ is a compact indecomposable object in $\mathcal{T}$ such that $M \nsubseteq \Sigma^{i} S$ for any $i$, then there exists a non-trivial ghost out of $M$.

Corollary 2.4. Let $\mathcal{T}$ be a triangulated category which admits arbitrary coproducts and let $S$ be a distinguished object. Every ghost in $\mathcal{T}$ is trivial if and only if $\mathcal{T}$ is the collection of retracts of coproducts of suspensions of $S$.

In the next section, we use these corollaries to determine when the stable module category of a finite $p$-group has no non-trivial ghosts. In the following section, we do the same for the derived category of a commutative ring.

\section{Stable module CATegories}

We begin with some preliminaries. Let $G$ be a finite group and let $k$ be a field. The stable module category $\operatorname{StMod}(k G)$ of $G$ is the category obtained from the category of left $k G$-modules by killing off the projectives. The space of morphisms from $M$ to $N$ in $\operatorname{StMod}(k G)$ is denoted $\underline{\operatorname{Hom}}_{k G}(M, N)$ and consists of the $k G$-module homomorphisms modulo those that factor through a projective module. Thus a 
map in the stable module category is trivial if and only if it factors through a projective module. A key fact [2] is that the Tate cohomology groups can be described as groups of morphisms in $\operatorname{StMod}(k G): \widehat{H}^{i}(G, M) \cong \underline{\operatorname{Hom}}\left(\Omega^{i} k, M\right) \cdot \operatorname{StMod}(k G)$ has the structure of a tensor triangulated category, where the trivial representation $k$ is the unit object and $\Omega$ is the loop (desuspension) functor. ( $\Omega M$ is defined to be the kernel of a projective cover of $M$. This is well-defined in the stable module category.) We denote by $\widetilde{\Omega}^{i}(M)$ the projective-free part of $\Omega^{i} M$, which is a welldefined $k G$-module. For more facts about $k G$-modules and $\operatorname{StMod}(k G)$, we refer the reader to Carlson's excellent lecture notes [4].

From now on we work exclusively with finite $p$-groups and assume that the characteristic of $k$ is $p$. We begin by proving the easy direction of our main theorem.

Proposition 3.1. If $G$ is either $C_{2}$ or $C_{3}$, then $\operatorname{StMod}(k G)$ has no non-trivial ghosts.

Proof. The group rings $k C_{2} \cong k[x] /\left(x^{2}\right)$ and $k C_{3} \cong k[x] /\left(x^{3}\right)$ are Artinian principal ideal rings. It is a fact $[9$, p. 170] that every module over an Artinian principal ideal ring is a direct sum of cyclic modules. We will use this fact to show that every projective-free $k G$-module is a direct sum of suspensions of $k$. The result will then follow from Corollary 2.4.

First consider the group $C_{2}$. By the above fact, we know that every module over the ring $k[x] /\left(x^{2}\right)$ is a direct sum of copies of $k$ and $k[x] /\left(x^{2}\right)$. In particular, every projective-free $k[x] /\left(x^{2}\right)$-module is a direct sum of copies of $k$. Now consider the group $C_{3}$. In this case, the above fact implies that every projective-free $k[x] /\left(x^{3}\right)$ module is a direct sum of copies of $k$ and $k[x] /\left(x^{2}\right)$. But note that

$$
\widetilde{\Omega}(k):=\operatorname{ker}\left(k[x] /\left(x^{3}\right) \rightarrow k\right) \cong k[x] /\left(x^{2}\right) .
$$

In both cases we have shown that every projective-free $k G$-module is a direct sum of suspensions of $k$. So we are done.

We now collect some facts about finite $p$-groups that we need in the sequel.

Lemma 3.2. Let $G$ be a finite $p$-group and let $M$ be a finite-dimensional nonzero $k G$-module. Then the invariant submodule $M^{G}$ of $M$ is non-zero. Thus there is only one simple $k G$-module, namely the trivial module $k$. Moreover, if $M^{G}$ is one-dimensional, then $M$ is indecomposable.

Proof. The proof of the first statement is an easy exercise; see [1, 3.14.1]. For the last statement, suppose to the contrary that $M \cong A \oplus B$, with $A$ and $B$ non-zero. Then we have that $M^{G} \cong A^{G} \oplus B^{G}$. This shows that $M^{G}$ is at least two-dimensional, for by the first part of the lemma, both $A^{G}$ and $B^{G}$ are at least one-dimensional. This contradiction completes the proof.

Lemma 3.3. Let $G$ be a finite p-group and let $I$ be a non-trivial, proper ideal of $k G$. Then $I$ is an indecomposable projective-free $k G$-module. In particular, the powers $J^{i}(k G)$ which are non-zero are indecomposable projective-free $k G$-modules.

Proof. We first show that $I$ is projective-free. If $I$ has a projective submodule, then since projective modules over finite $p$-groups are free, that would mean that $I$ should have $k$-dimension at least $|G|$, which is not possible since $I$ is proper. To prove that $I$ is indecomposable, it suffices to show (by Lemma 3.2) that $I^{G}$ is 
one-dimensional. Note that $I^{G}=I \cap(k G)^{G}$. It is easy to see that $(k G)^{G}$ is the onedimensional subspace generated by the norm element $\sum_{g \in G} g$. We also know from Lemma 3.2 that $I^{G}$ is non-zero. Therefore $I^{G}$ is a one-dimensional submodule.

Lemma 3.4. Let $G$ be a finite p-group. Then, for all integers $i$, we have

$$
\operatorname{dim}\left(\widetilde{\Omega}^{i} k\right) \equiv(-1)^{i} \bmod |G| .
$$

Proof. Recall that $\widetilde{\Omega}^{1} k$ is the kernel of the augmentation map, so we have a short exact sequence

$$
0 \longrightarrow \widetilde{\Omega}^{1} k \longrightarrow k G \longrightarrow k \longrightarrow 0,
$$

which tells us that $\operatorname{dim}\left(\widetilde{\Omega}^{1} k\right) \equiv-1$ modulo $|G|$. Inductively, it is clear from the short exact sequences

$$
0 \longrightarrow \widetilde{\Omega}^{i+1} k \longrightarrow(k G)^{t} \longrightarrow \widetilde{\Omega}^{i} k \longrightarrow 0
$$

that $\operatorname{dim}\left(\widetilde{\Omega}^{i} k\right) \equiv(-1)^{i}$ modulo $|G|$ for $i \geq 0$. (Here $(k G)^{t}$, for some $t$, is a minimal projective cover of $\widetilde{\Omega}^{i} k$.) Also, since $\widetilde{\Omega}^{i} k \cong\left(\widetilde{\Omega}^{-i} k\right)^{*}$ in $\operatorname{Mod}(k G)$, it follows that $\operatorname{dim}\left(\widetilde{\Omega}^{i} k\right) \equiv(-1)^{i}$ modulo $|G|$ for each integer $i$.

We now introduce a formula of Jennings. Let $G$ be a finite $p$-group and let $J(k G)$ be the Jacobson radical of $k G$. Since $k G$ is a local Artinian ring, it follows that $J(k G)$ is nilpotent. So there exists a smallest integer $m$ such that $J(k G)^{m}=0$. This integer will be called the nilpotency index of $J(k G)$, and it can be shown to be independent of the field $k$. Very closely related to the powers of the Jacobson radical are the dimension subgroups of $G$, which we now define. The dimension subgroups $F_{i}$ of $G$ are defined by

$$
F_{i}:=\left\{g \in G: g-1 \in J^{i}(k G)\right\} \quad \text { for } i \geq 1 .
$$

These form a descending chain of normal subgroups in $G$

$$
F_{1} \supseteq F_{2} \supseteq F_{3} \supseteq \cdots \supseteq F_{d} \supseteq F_{d+1},
$$

with $F_{1}=G$ and $F_{d+1}$ trivial. Define integers $e_{i}$ by $p^{e_{i}}=\left[F_{i}: F_{i+1}\right]$ for $1 \leq i \leq d$. Then a formula due to Jennings [7] states that the nilpotency index $m$ of $J(k G)$ is given by

$$
m=1+(p-1) \sum_{i=1}^{d} i e_{i} .
$$

Moreover, $e_{1}$ is the minimal number of generators for $G$. From the definition of the numbers $e_{i}$, it is clear that $|G|=p^{\sum_{i} e_{i}}$.

Proposition 3.5. Let $G$ be a non-trivial finite p-group that is not isomorphic to $C_{2}$ or $C_{3}$. Then there exists a finite-dimensional indecomposable projective-free $k G$-module that is not isomorphic to $\widetilde{\Omega}^{i} k$ for any $i$. In particular, there exists a non-trivial ghost in $\operatorname{StMod}(k G)$.

Proof. It is well known that there are indecomposable projective-free modules over the Klein four group $\left(C_{2} \oplus C_{2}\right)$ which are not of the form $\widetilde{\Omega}^{i}(k)$. In fact, every evendimensional projective-free indecomposable $k\left(C_{2} \oplus C_{2}\right)$-module has this property. Such modules are known to exist; see [1, Thm. 4.3.3], for instance. Therefore, we can assume that $G$ is not any one of the groups $C_{2}, C_{3}$ and $C_{2} \oplus C_{2}$. 
Consider the module $J^{2}(k G)$, which we denote by $J^{2}$ for brevity. We will show below that $J^{2} \neq 0$. Then, by Lemma 3.3, we know that $J^{2}$ is an indecomposable projective-free $k G$-module. Let $|G|=p^{n}$ for some positive integer $n$. Since the dimension of $\widetilde{\Omega}^{i} k$ is +1 or -1 modulo $|G|=p^{n}$ (see Lemma 3.4), we will be done if we can show that the congruence class $\bmod p^{n}$ of $\operatorname{dim}\left(J^{2}\right)$ is different from +1 and -1 . In fact, we will show that when $G$ is not one of the above 3 groups, then

$$
1<\operatorname{dim}\left(J^{2}\right)<p^{n}-1 .
$$

Note that $J^{2} \subsetneq J$. For, otherwise, Nakayama's lemma would imply that $J=0$, a contradiction. Therefore the second inequality is clear. Now we establish the first inequality. We have two cases to consider here:

Case 1: Suppose $\operatorname{dim} J^{2}=0$. Then the nilpotency index $m$ of $J$ is 2 . So by Jennings' formula we have

$$
2=1+(p-1)\left[e_{1}+2 e_{2}+\cdots+d e_{d}\right] .
$$

This means $1=(p-1)\left[e_{1}+2 e_{2}+\cdots+d e_{d}\right]$. Recall that $e_{1}$ is the minimal number of generators of $G$, so $e_{1}>0$. Therefore, the last equation holds if and only if $p=2$, $e_{1}=1$ and $e_{i}=0$ for $i \geq 2$. Since $|G|=p^{\sum_{i} e_{i}}$, it follows that $J^{2}=0$ if and only if $G=C_{2}$. But $G \neq C_{2}$, by assumption. So this case cannot arise.

Case 2: Suppose $\operatorname{dim} J^{2}=1$. That means $J^{2}=k$, therefore $J\left(J^{2}\right)=J(k)=0$. So the nilpotency index $m$ of $J$ is 3 . By Jennings' formula we have

$$
3=1+(p-1)\left[e_{1}+2 e_{2}+\cdots+d e_{d}\right] .
$$

This means $2=(p-1)\left[e_{1}+2 e_{2}+\cdots+d e_{d}\right]$. Here there are two possibilities. Either $p=3, e_{1}=1$, and $e_{i}=0$ for all $i \geq 2$, or $p=2, e_{1}=2$, and $e_{i}=0$ for all $i \geq 2$. In the former, we have $|G|=3$, so $G \cong C_{3}$, and in the latter, $|G|=4$ and $G$ is generated by 2 elements, so $G \cong C_{2} \oplus C_{2}$. But $G$ was assumed to not be one of these groups, so this case also cannot arise.

Since both cases are ruled out, we have proved the first inequality.

Finally, the last statement of the proposition follows from Corollary 2.3.

The main step of the proof of Proposition 3.5 is essentially the classification of $p$-groups with nilpotency index at most 3 . This is known to be an easy consequence of Jennings' formula, but we have included a proof to keep the paper self-contained.

Combining Propositions 3.1 and 3.5, we have proved our main theorem:

Theorem 3.6. Let $G$ be a non-trivial finite p-group and let $k$ be a field of characteristic $p$. Then $\operatorname{StMod}(k G)$ has no non-trivial ghosts if and only if $G$ is either $C_{2}$ or $C_{3}$.

We extract the following interesting result from the proof of Proposition 3.5.

Proposition 3.7. Let $G$ be a non-trivial finite p-group. Then $J^{2}(k G) \cong \widetilde{\Omega}^{i} k$ in $\operatorname{Mod}(k G)$ for some $i$ if and only if $G$ is isomorphic to $C_{3}$ or $C_{2} \oplus C_{2}$.

Proof. The only if part follows from the proof of Proposition 3.5. For the converse, if $G$ is $C_{3}$, then every finite-dimensional indecomposable projective-free module (and hence $J^{2}(k G)$ ) is of the form $\widetilde{\Omega}^{i} k$, for some $i$; see the proof of Proposition 3.1. If $G$ is $C_{2} \oplus C_{2}$, then $J^{2}(k G) \cong k$. This completes the proof of the proposition. 


\section{Derived CATEgories}

Let $R$ be a commutative ring and let $D(R)$ be its (unbounded) derived category. This has the structure of a tensor triangulated category with $R$ (viewed as a chain complex concentrated in degree zero) as the unit object. Observe that a map $\phi: X \rightarrow Y$ in $D(R)$ is a ghost if and only if the induced map $H_{*}(\phi): H_{*}(X) \rightarrow$ $H_{*}(Y)$ in homology is zero. The natural question is to characterise commutative rings $R$ for which $D(R)$ has no non-trivial ghosts. This has been done by Lockridge in [8]. We include a proof below for the reader's convenience and also to illustrate the results in Section 2.

Theorem 4.1 ([8]). Let $R$ be a commutative ring. Then $D(R)$ has no non-trivial ghosts if and only if $R$ is a finite product of fields.

Proof. Let $R$ be a finite product of fields, $F_{1} \times F_{2} \times \cdots \times F_{l}$, say. Then every $R$ module splits naturally as a direct sum of modules over the subrings $F_{i}$. It follows that $D(R)$ is equivalent to $D\left(F_{1}\right) \times D\left(F_{2}\right) \times \cdots \times D\left(F_{l}\right)$. Now note that the derived category of a field $F$ is equivalent to the category of $\mathbb{Z}$-graded $F$-vector spaces. From this it follows that every object in $D(R)$ is a retract of direct sum of suspensions of $R$. Therefore, by Corollary 2.4, $D(R)$ does not have any non-trivial ghosts. Conversely, suppose there are no non-trivial ghosts in $D(R)$. Then it is not hard to see that for every pair of $R$-modules $M$ and $N$, we have $\operatorname{Ext}_{R}^{i}(M, N)=0$ for each $i>0$. This implies that every $R$-module is projective. Therefore $R$ is semi-simple; see $[10, T h m .4 .2 .2]$. Since commutative semi-simple rings are precisely finite direct products of fields (by the Artin-Wedderburn theorem), we are done.

The answer to the riddle posed in the introduction should be now clear to the reader. The only non-trivial finite $p$-groups that are like a finite product of fields are $C_{2}$ and $C_{3}$.

\section{REFERENCES}

[1] D. J. Benson. Representations and cohomology. I, volume 30 of Cambridge Studies in Advanced Mathematics. Cambridge University Press, Cambridge, 1998. MR1644252 (99f:20001a)

[2] D. J. Benson and Jon F. Carlson. Products in negative cohomology. J. Pure Appl. Algebra, 82(2):107-129, 1992. MR1182934 (93i:20058)

[3] David Benson, Sunil K. Chebolu, J. Daniel Christensen, and Ján Mináč. The generating hypothesis for the stable module category of a p-group. Journal of Algebra, 310(1):428-433, 2007. MR2307802 (2007k:16011)

[4] Jon F. Carlson. Modules and group algebras. Lectures in Mathematics ETH Zürich. Birkhäuser Verlag, Basel, 1996. Notes by Ruedi Suter. MR1393196 (97c:20013)

[5] J. Daniel Christensen. Ideals in triangulated categories: phantoms, ghosts and skeleta. Adv. in Math., 136:284-339, 1998. MR1626856 (99g:18007)

[6] Peter Freyd. Stable homotopy. In Proc. Conf. Categorical Algebra (La Jolla, Calif., 1965), pages 121-172. Springer, New York, 1966. MR0211399 (35:2280)

[7] S. A. Jennings. The structure of the group ring of a $p$-group over a modular field. Trans. Amer. Math. Soc., 50:175-185, 1941. MR0004626 (3:34f)

[8] Keir Lockridge. The generating hypothesis in the derived category of $R$-modules. Journal of Pure and Applied Algebra, 208(2):485-495, 2007. MR2277690 
[9] D. W. Sharpe and P. Vámos. Injective modules. Cambridge University Press, London, 1972. MR0360706 (50:13153)

[10] Charles A. Weibel. An introduction to homological algebra, volume 38 of Cambridge Studies in Advanced Mathematics. Cambridge University Press, Cambridge, 1994. MR1269324 (95f:18001)

Department of Mathematics, University of Western Ontario, London, Ontario, CANADA

E-mail address: schebolu@uwo.ca

Department of Mathematics, University of Western Ontario, London, Ontario, Canada

E-mail address: jdc@uwo.ca

Department of Mathematics, University of Western Ontario, London, Ontario, CANAdA

E-mail address: minac@uwo.ca 